\title{
Transatlantica
}

Revue d'études américaines. American Studies Journal

\section{Mieux comprendre ce que fait la droite ultra aux} Etats-Unis

Compte rendu des livres de Lewis Lapham, Serge Halimi, Scott Lucas et Thomas Frank

\section{Pierre Guerlain}

\section{(2) OpenEdition} Journals

Édition électronique

URL : http://journals.openedition.org/transatlantica/847

DOI : $10.4000 /$ transatlantica.847

ISSN : $1765-2766$

Éditeur

AFEA

Référence électronique

Pierre Guerlain, « Mieux comprendre ce que fait la droite ultra aux Etats-Unis », Transatlantica [En ligne], 1 | 2005, mis en ligne le 22 avril 2006, consulté le 29 avril 2021. URL : http://journals.openedition.org/ transatlantica/847 ; DOI : https://doi.org/10.4000/transatlantica.847

Ce document a été généré automatiquement le 29 avril 2021.

\section{(c)}

Transatlantica - Revue d'études américaines est mis à disposition selon les termes de la licence Creative Commons Attribution - Pas d'Utilisation Commerciale - Pas de Modification 4.0 International. 


\title{
Mieux comprendre ce que fait la droite ultra aux Etats-Unis
}

\author{
Compte rendu des livres de Lewis Lapham, Serge Halimi, Scott Lucas et \\ Thomas Frank
}

Pierre Guerlain

1 Lewis Lapham, Theater of War, In Which the Republic Becomes an Empire, New York, 2002, 2003, The New Press, 278 p., 14.95 \$

2 Lewis Lapham, Gag Rule, On the suppression of Dissent and the Stifling of Democracy, New York, The Penguin Press, 2004, 178 p., 19.95 \$

3 Serge Halimi, Le Grand bond en arrière, Comment l'ordre libéral s'est imposé au monde, Paris Fayard, 2004, 620 p., 23 euros

4 Scott Lucas, The Betrayal of Dissent, Beyond Orwell, Hitchens and The New American Century, London, Pluto Press, 2004, 324 p., 10,99£

5 Thomas Frank, What's the Matter with Kansas? How Conservatives Won the Heart of America, Metropolitan Books, Henry Holt and Company, New York 2004, 306 pp, 24 \$ (édition utilisée ici: What's The Matter with America? The Resistible Rise of the American Right, Secker and Warburg, Londres, 2004, 306 p., 12£)

6 Ces cinq livres sont fort différents mais traitent tous, totalement ou en grande partie, des évolutions politiques et idéologiques aux Etats-Unis dans la période récente, c'est à dire depuis l'arrivée au pouvoir de l'administration Bush. Ils posent aussi le problème des relations entre journalisme et travail universitaire. Lewis Lapham est journaliste, il écrit une tribune mensuelle dans le magazine Harper's et le premier ouvrage sélectionné ici est un recueil de ses tribunes publiées entre Octobre 2000 et juin 2003 alors que le second est un ouvrage sur les restrictions des libertés publiques aux EtatsUnis. On verra que le journaliste a vu plus juste - et plus tôt - que de nombreux politistes ou politologues universitaires englués dans leur respect de la parole du pouvoir. Lapham a un style vif et écrit dans un très bel anglais, sa qualité de langue évoque pour moi la beauté de l'allemand de Freud. C'est en écrivain et styliste qu'il 
commente, sur un ton parfois désabusé, les bizarreries ou mensonges des pouvoirs en place.

7 On est frappé de voir qu'avec très peu de recul Lapham, sur la base d'une fine analyse des discours officiels, comprend tout de suite les implications politiques des diverses décisions ou discours de l'Administration. Sa tribune de décembre 2002 s'intitule « Hail Caesar ", ce qui ne manquera pas de provoquer les sarcasmes des politistes patentés. Il cite Abraham Lincoln pour déconstruire ce qui est déjà le mensonge de $\mathrm{M}$. Bush quant à l'Irak: « Allow the President to invade a neighboring country whenever he shall deem it necessary to repel an invasion...and you allow him to make war at pleasure » déclarait Lincoln. La suite des événements en Irak en 2003 a montré que le Congrès américain n'a pas joué son rôle de contrepoids et a effectivement laissé le président faire la guerre à sa guise. De façon générale, Lapham montre qu'il est très cultivé et qu'il sait citer les auteurs qui permettent le mieux une mise en lumière des politiques américaines. Sa culture n'est pas uniquement politique, il fait référence à des philosophes, des historiens des écrivains. Il est clair que Lapham est quelqu'un qui connaît ses humanities et que sa grande culture lui permet de saisir les non-dits des discours officiels. Le lire est un plaisir et une leçon de lucidité.

Lors d'une réunion à l'Institut d'Etudes Politiques en juin 2002 où j'ai fait remarquer à un spécialiste américain de politique étrangère, John Ikenbury, auteur de talent, que la thèse des armes de destruction massive ("weapons of mass destruction ») était plutôt de « weapons of mass deception ». Il m'a rétorqué qu'en Europe, on avait trop tendance à rejeter tout ce que disait le président américain car ce dernier, au contraire de Clinton, n'était pas populaire mais que l'on avait tort. Lewis Lapham, journaliste talentueux, comprenait mieux la situation que le spécialiste de politique étrangère. Nos collègues qui utilisent le mot «journaliste " comme insulte dévalorisante devraient songer à ce genre de situation. Le grand spécialiste des relations internationales Francois Heisbourg avait prédit que la France rallierait le camp américain le temps venu. Un grand nombre de politologues français étaient alignés sur une ligne blairiste, qu'ils pensaient médiane entre celles de Bush et de Chirac. Les erreurs d'alors n'ont pas été suivies d'explications par la suite. On aimerait que les politologues "scientifiques " qui se sont trompés sur l'Afghanistan ou qui ont naïvement cru au « conservatisme de compassion" avancé par M. Bush dans sa campagne de 2000, fassent ce que les scientifiques des sciences dures sont contraints de faire : qu'ils reconnaissent que leurs théories se sont révélées erronées et amendent celles-ci en conséquence. Un exercice salutaire consisterait à confronter les analyses dix ans après leurs formulations initiales, la scientificité de la science politique prendrait quelques coups.

Dans Gag Rule Lapham offre une analyse non seulement des attaques visant la liberté d'expression aux Etats-Unis mais aussi de la servilité des médias et de la plupart des élites intellectuelles et culturelles face au gouvernement qui les prive de libertés démocratiques. On voit ainsi le Congrès encore une fois ne pas jouer son rôle constitutionnel et accepter en silence les assauts liberticides de MM Ashcroft et Bush ( «No points of order or objection, no memorable speech, nothing but the steady murmur of approval and the quiet hum of praise », p 5). Lapham sait aussi que la situation actuelle n'est pas totalement inédite; ainsi fait-il référence à l'histoire grecque en 416 av JC lors de la guerre entre Athènes et Sparte pour éclairer l'aventure américaine en Irak (la justice n'existe qu'entre égaux, sinon les forts font ce qu'ils veulent et les faibles se soumettent). Parlant des si mal nommés «néo-conservateurs ", 
qu'il désigne des termes de "utopian anarchists » ou " radical nationalists ", il évoque l'Inquisition espagnole et son injonction aux individus accusés d'hérésie: "Profess your faith in us or we will burn you at the stake. » Il donne aussi une citation de Göring lors du procès de Nuremberg: "All you have to do is tell them that they're being attacked, denounce the pacifists for lack of patriotism and exposing the country to danger. It works the same in any country. » Lapham ne dit pas que Bush est un nazi, mais qu'il utilise une technique de manipulation déjà utilisée auparavant par les nazis, ce qui est différent mais pas très rassurant. Certes, des historiens méticuleux pourront montrer que les Etats-Unis ne sont pas l'Espagne de l'Inquisition, que citer Göring pour dénoncer les mensonges de $\mathrm{M}$. Bush établit une analogie problématique, mais le lecteur doit comprendre que la citation d'un ennemi officiellement abhorré doit faire choc pour faire saisir le non dit.

Deux beaux et bons livres qui permettent de s'oxygéner les méninges et dont l'écriture n'est pas aussi aride que celle des dissidents plus connus. Les deux suivants sont des livres écrits par des universitaires dont l'un, Serge Halimi, qui a enseigné aux EtatsUnis et en France, choisit ici de se présenter comme journaliste. Ce politologue ne partage donc pas les préventions de ses collègues vis à vis du journalisme. Ce qui importe ici est de savoir si l'on a à faire à un discours de vérité bien informé ou pas. Le statut professionnel importe peu quant à la qualité de la recherche. Halimi a écrit un livre fort documenté qui est aussi militant et rageur. Comme son titre l'indique, il cherche à retracer le parcours des idées "néo-libérales " pensées par Friedrich von Hayek puis mises en pratique par tous les gouvernements d'Amérique et d'Europe (et de quelques autres lieux). Il s'intéresse à divers pays dont la France et la Nouvelle Zélande dont je ne parlerai pas ici.

11 Son livre est tout à fait intéressant pour tous les américanistes qui s'intéressent à l'économie, aux idéologies et au politique. Il retrace ainsi l'histoire des boites à idées «néo-libérales » ou « néo-conservatrices » américaines, ainsi que celles des discours et pratiques de l'Administration Reagan. Il utilise les travaux de très nombreux auteurs américains, souvent des universitaires (par exemple, Lasch, Lind, Frank, Chomsky, Said) et met en rapport déclarations et réalités, il pointe les décalages entre divers moments de la carrière politique de divers acteurs de la scène politique. Halimi est, certes, un homme en colère qui n'hésite pas à nommer ses confrères politologues qui se contredisent ou dont la science se perd dans les croyances idéologiques. On peut ne pas être d'accord ici ou là, regretter son ton ou ses présentations parfois partielles donc partiales. Mais alors que la pauvreté s'aggrave, que les Etats-Unis s'embourbent en Irak et alourdissent leurs déficits et alors que les salaires des dirigeants d'entreprise s'envolent et que les millionnaires accaparent une part toujours plus grande des richesses du pays, on peut comprendre la colère d'un chercheur qui est aussi citoyen. A lire Halimi, on se dit que la social-démocratie d'antan est presque révolutionnaire, car aujourd'hui penser que tout le monde a droit à un système de santé efficace, une éducation correcte, à un emploi payé convenablement est déjà se placer hors du mainstream idéologique.

Ce livre fourmille de références utiles pour tous ceux qui s'intéressent à la vie politique des Etats-Unis. Il permet aussi de réfléchir à ce qu'est un ouvrage militant. Halimi annonce clairement la couleur, alors qu'un Huntington ou un Adjami, un Fukuyama ou même un Nye, sont des militants qui ne disent pas d'où ils parlent, qui laissent croire que leur science politique est neutre, qui font mine de parler au nom de la science alors 
qu'ils le font aussi au nom d'une idéologie et pour des groupes ou des institutions spécifiques. L'opposition binaire entre chercheurs et militants, ou entre scientifiques et journalistes doit être complexifiée et pensée en termes intellectuels.

Scott Lucas est britannique, professeur de civilisation américaine à Birmingham, et il s'intéresse ici aux campagnes de dénigrement visant les contestataires. Son livre établit des liens entre divers auteurs, Orwell et Hitchens, par exemple, et étudie le rôle qu'ils ont joué dans la décrédibilisation de la gauche. Orwell, on le sait maintenant, a donné aux services secrets britanniques, une liste de compagnons de route du Parti communiste au tout début de la guerre froide. Lucas cependant s'intéresse surtout à la façon dont Orwell a été canonisé par la droite dans sa lutte anticommuniste. Le cas d'Orwell est complexe et cet auteur a produit une œuvre critique qui reste forte aujourd'hui. Cependant, ce qui nous intéresse ici en tant qu'américanistes est l'analyse de la décrédibilisation des contestataires américains. Scott Lucas est un grand lecteur de journaux américains et un connaisseur des émissions de télévision tant américaines que britanniques. Il analyse la construction de ce que l'on pourrait appeler la nouvelle censure, celle qui passe par la disparité des moyens d'information à la disposition des divers courants idéologiques mais aussi les utilisations récentes des vieilles techniques de censure (intimidation des opposants par le porte-parole de la Maison Blanche, intimidation des manifestants ou des membres d'associations critiques du gouvernement, boue lancée contre tous ceux qui osent élever la voix, même s'il s'agit de gens aussi peu gauchistes que Geoge Soros, autrefois surtout connu pour sa spéculation contre la livre sterling, création d'une agence officielle de désinformation, le fameux Office of Strategic Influence créé par le Ministère de la Défense, qui est ensuite abolie - mais est-ce la désinformation qui commence ?). Au passage, Scott Thomas montre que l'information sur les Etats-Unis et leur politique étrangère dans les grands médias est plus complète en Grande-Bretagne qu'aux Etats-Unis même. Comparer la BBC et la Fox ou The Guardian et le New York Times est assez instructif sur ce plan. Ce livre poursuit le travail de Chomsky et Herman sur la fabrication du consensus (manufacture of consent), à partir de données qui concernent une période différente.

Enfin le dernier livre, celui de Thomas Frank au sous-titre inspiré de Brecht traite, pour les Etats-Unis, du même problème que celui d'Halimi, à savoir la montée des idées de droite. La méthode de ce journaliste, docteur de l'université, est cependant assez différente. L'auteur parle surtout du Kansas, un état qu'il connaît bien pour y avoir passé son enfance et traite du Kansas comme d'une métonymie des Etats-Unis. Frank cherche à comprendre comment même un état aux traditions populistes de gauche comme le Kansas a pu basculer dans l'escarcelle du parti républicain. Surtout, il analyse l'évolution idéologique d'une partie de ce que l'on pourrait encore appeler en Europe la " classe ouvrière ». Il analyse comment ces ouvriers ont pu conserver leur rhétorique populiste en intégrant le parti républicain et en former l'aile la plus dure. Le parti républicain est maintenant divisé entre les "con men" (conservative men) et le "mod squad» (moderates). Les "modérés", ici, sont les hommes d'affaire qui ne sont ni homophobes, ni chrétiens fondamentalistes, mais n'ont plus rien de keynésiens, et ne sont surtout pas des sociaux-démocrates à l'européenne. On l'aura compris, le combat pour les valeurs est passé par là. La lutte contre l'avortement, contre les programmes scolaires jugés laxistes ou anti-chrétiens, lutte pour la patriotisme et l'amour de l'Amérique contre les subversifs de l'ACLU a occupé le terreau populiste et transformé les batailles politiques américaines. Frank et Halimi sont très proches dans leurs analyses du parti démocrate : celui-ci s'est déplacé à droite, a abandonné tout discours 
de défense des travailleurs et des syndicats, discours que l'on appelait «lutte des classes » dans le langage marxiste qui était celui de la gauche européenne. Le parti démocrate s'est lui surtout intéressé aux voix de la classe moyenne et aux possibilités de financement des campagnes électorales par les grandes entreprises. Le vide créé à gauche a profité à une ultra droite moralisatrice qui vote républicain aux Etats-Unis. On peut voir en France un équivalent structurel dans la montée du Front national (ou du NPD en Allemagne).

15 La droite extrême américaine a su s'inspirer des théories de Gramsci et créé une coalition très hétéroclite sur le plan des valeurs entre, d'une part, des entrepreneurs capitalistes modernes, favorables à la mondialisation et aux délocalisations et hostiles aux discriminations ethniques ou sexuelles et, d'autre part, une partie des classes défavorisées qui craignent le chômage, les délocalisations et s'accrochent à des formes de religiosité anti-modernistes. La lutte des classes s'est déplacée vers les luttes intestines du parti républicain, et Frank note avec une ironie mordante que les nouveaux républicains conservateurs votent systématiquement pour les responsables qui mettent leur survie économique en danger. La lutte anticommuniste avait déjà permis de fédérer des groupes aux intérêts fort divergents, la lutte pour les valeurs chrétiennes et/ou américaines autorise la création d'un même amalgame. Les " angry white men » du Kansas et d'ailleurs ne considèrent pas le Big Business comme leur ennemi mais choisissent plutôt le féminisme, les universitaires, les terroristes, les humanistes séculiers et tout un tas d'autres démons, évidemment créés à des fins partisanes par les républicains dits " modérés ", donc par le Big Business. La droite avait déjà réussi sa stratégie de division ethnique (la « southern strategy » de Nixon); elle est en passe de réussir sa mystification par référence au combat pour les «valeurs américaines ». Thomas Frank offre une analyse convaincante de ces phénomènes qui touchent l'Europe également sous d'autres formes.

Frank avait, dans un autre ouvrage, One Market Under God, brocardé certains universitaires épris de théories regroupées de façon problématique sous l'étiquette de cultural studies qui ne s'intéressaient qu'au texte et pas au contexte, et dont les catégories d'analyse recoupaient celles de la publicité (la résistance dans les clips de Madona, les batailles sémantiques qui laissent de côté les enjeux matériels, le nonréférentialisme....). On peut se demander, comme l'a fait Gitlin dans The Twilight of Common Dreams, et comme le fait ici Halimi, si l'université n'a pas sa part de responsabilité dans les dérives fondamentalistes et droitières, qui se font jour des deux côtés de l'Atlantique. L'ethnicisation des cultural studies a gommé les enjeux communs à tous et ringardisé les discours en termes de classes sociales. Le multiculturalisme extrême a donc, sans s'en rendre compte, mordu à l'hameçon nixonien puis reaganien. Reagan n'a pas aboli les programmes d'affirmative action qu'il disait abominer. Au contraire, ces programmes, qui ont eu un effet minime de tokenism, lui ont permis de maintenir des divisions entre groupes appartenant aux mêmes classes sociales mais divisés par de subtiles instrumentalisations du racisme.

17 Parfois, l'université, en voulant gommer tout racisme, a sombré dans ce que Bourdieu a appelé un racisme social visant précisément ces catégories sociales aujourd'hui devenues populistes de droite ou d'extrême droite. En abandonnant toutes formes d'universalisme, l'université a favorisé les discours et pratiques séparatistes en son sein, mais aussi dans la société dans son ensemble. Les groupes les plus faibles, y compris ceux que l'université voulait mieux intégrer, c'est à dire les minorités, sont 
ceux qui souffrent le plus des révolutions conservatrices qui de Reagan à Gingrich et Bush I et II s'abattent sur les Etats-Unis. La part de l'université n'est évidemment pas prépondérante et il ne s'agit en aucun cas de sous-estimer l'inventivité des stratèges républicains de Atwater à Rove, ni d'oublier les facteurs historiques qui expliquent la relégation des minorités comme des pauvres. Frank, comme Halimi ou Lasch, note cependant le déclin des discours rassembleurs au-delà de l'ethnicité et en tant qu'américanistes et universitaires, il me semble que nous devrions ouvrir un chantier de recherches portant sur la réduction ethnique des luttes politiques et sociales, sur la pan-ethnicisation qui a saisi le discours médiatique et accompagné le reflux de la gauche. La nouvelle gauche des années 60 puis 70 a mis l'accent sur des phénomènes négligés par la vieille gauche, ce qui a eu des effets positifs et conduit à l'éclosion du féminisme, des ethnic studies, des gender studies, puis des queer studies, puis, par la suite elle a liquidé les problématiques de classes avant d'être elle-même exclue de l'espace public et de se réfugier à l'université.

La lutte contre l'universalisme hérité des Lumières a pu, tout d'abord, sembler libératrice car l'universel, né en Occident, était local, mais une fois l'universalisme terrassé sur le plan idéologique, il n'est plus resté d'idéologie mobilisatrice à la gauche et le localisme ethnique n'a pas pu résister aux assauts universalistes religieux des fondamentalistes, qui sont devenus un groupe pivot dans le parti républicain et donc dans la société américaine. La nouvelle gauche a certes créé de nouveaux champs d'étude et d'intervention, mais au lieu de simplement combler les oublis de l'ancienne gauche, elle a aussi inventé de nouvelles amnésies et n'a pas pris en compte le contexte politique dans lequel elle opérait.

De ce qui précède, il ne faudra pas tirer la conclusion que l'étude de l'ethnicité n'a aucun intérêt, ni que l'université n'a rien produit de stimulant. Comme il a été dit par Todorov, lors du colloque organisé sur l'œuvre d'Edward Said à la bibliothèque Mitterrand à Paris les 24 et 25 septembre, Said n'a jamais renié les Lumières ni le lien entre mots et monde; il était opposé à tout enfermement ethnique. Il n'a jamais déplacé les enjeux du politique vers le pur sémantique et le pur symbolique (et pour cause!). C'est donc plutôt vers Said, Judith Butler ou encore Henry Louis Gates et William Julius Wilson que l'on peut se tourner pour éviter la pan-ethnisiation et le déni de prise en compte des formes d'injustice qui ne visent pas explicitement les minorités (ethniques ou sexuaelles). Les penseurs du séparatisme ethnique, voire sexuel, ont mis la nouvelle gauche dans une impasse et favorisé, à leur corps défendant, bien sûr, la montée du populisme d'extrême droite. Ce que ces divers livres montrent c'est, d'une part, que certains Américains pensent clairement les dérives des dirigeants de leur pays et échappent à la doxa droitière et, d'autre part, qu'un nouveau rassemblement multiethnique est nécessaire entre tous les exclus du fonctionnement économique et politique mis en place par les révolutionnaires de la droite. Oui, la majorité compte, la majorité qui, des pauvres à la classe ouvrière et à la majeure partie de la classe dite moyenne, n'ont rien à gagner en suivant les sirènes fondamentalistes, celles de la religion, comme celles du capitalisme qui aspire à redevenir sauvage en gommant tout le XXe siècle.

Voici donc cinq livres qui questionnent les orthodoxies, agacent aussi parfois et surprennent. Ils font tous une utilisation spécifique de l'histoire mais ne sont pas des ouvrages d'historiens. Les citoyens, les politistes, de toute évidence, ne peuvent pas bénéficier du recul historique pour penser l'événement. Il faut penser au vif et le faire 
en restant vif. Voilà ce qui rapproche ces livres assez divers sur le plan stylistique et qui traitent de phénomènes contemporains d'une importance capitale. Enfin pour ne pas sombrer dans le présentisme extrême, je propose de méditer une phrase de Christopher Lasch, publiée en 1979 dans The Culture of Narcissism : «Cultural radicalism has become so fashionable and so pernicious in the support it unwittingly provides for the status quo, that any criticism of contemporary society that hopes to get beneath the surface has to criticize, at the same time, much of what currently goes under the name of radicalism». Encore une opposition binaire à déconstruire, celle qui oppose « radicaux » et conservateurs.

INDEX

Thèmes : Recensions

AUTEUR

PIERRE GUERLAIN

Université Paris X Nanterre 